\title{
Quantum Mechanical Analysis of GaN Nanowire Transistor for High Voltage Applications
}

\author{
Neel Chatterjee, Sujata Pandey \\ Department of Electronics and Communication Engineering, \\ Amity University Uttar Pradesh, Noida-201313, India
}

(Received 30 August 2016; published online 23 December 2016)

\begin{abstract}
The paper presents the quantum mechanical analysis of Gallium Nitride nanowire transistor. The effect of high $k$ gate dielectric and different gate metals on the electrical characteristics of the device is studied. Nanowire width has been reduced to have a proper control on the device characteristics at reduced gate length. A high value of drain current is obtained at gate length of $10 \mathrm{~nm}$ and nanowire radius equal to $10 \mathrm{~nm}$, is obtained. Also high voltage operation of the device upto 40 volts has been shown. A flatter transconductance curve is obtained which shows the linearity of the device. The results obtained are comparable with the experimental and simulated data reported in literature
\end{abstract}

Keywords: Quantum mechanical, Nanowire, High $k$ gate dielectric.

DOI: $10.21272 /$ jnep.8(4(2)).04063

PACS numbers: 62.23. $\mathrm{Hj}, 85.30 . \mathrm{Tv}$, 07.05.Tp, 85.30. - z

\section{INTRODUCTION}

Cylindrical Nanowire FET is now gaining much traction in the research areas due to its unique structure and excellent subthreshold characteristics and scalability to different sizes along with noise reduction [1-7]. The reason for such improved performance is because of the structure of the gate which surrounds the channel completely and thus provides a better control over the current by the gate.

Gallium Nitride (GaN) has now been widely used for high electron mobility transistors (HEMT) due to its high mobility and also because of its wide bandgap which endows it the property of high breakdown voltage and allows it to be used for high voltage applications [8-9]. GaN nanowires have been in fabrication processes for a long time[10-11] and been used for photo-detection and photoluminescence applications [1213]. Extensive research has been done in the area of employing GaN to nanodevices and FET's [14-18]. AlGaN/GaN nanowire FET was studied in details by Kang et.al. [17] which showed a positive drift in threshold voltage at lower gate widths. Li et. al. [18], demonstrated experimentally $\mathrm{Ga}_{2} \mathrm{O}_{3} / \mathrm{GaN}$ nanowire transistor with $50 \mathrm{~nm}$ and $20 \mathrm{~nm}$ gate length where scaling issues and short channel effects were analysed. Chen et.al. [19], investigated on the electrical properties of individual GaN nanowire based ferroelectric FETs. Mussener et et. al. [20], studied the internal electric fields in AlGaN/GaN nanowire heterostructures. Zhao et. al. [21], proposed a low cost green method to synthesize GaN nanowires which could be effectively used in GaN based nanowire FETs. Also, Fang et.al. [22], incorporated Silicon donor in GaN nanowires and FET measurements combined with finite mobility of carriers in the non-intentionally doped nanowires were carried out.

Keeping in view of the wide applications of GaN nanowire transistors and with the devices reaching smaller dimensions quantum mechanical analysis of the device is desired for gate lengths below $20 \mathrm{~nm}$. The scaling down of the device gives rise to many effects which can only be accurately described by employing quantum mechanical model for analysis.

In this paper we propose quantum mechanical analysis of sub-20 nm GaN nanowire FET for high voltage applications. The device has been simulated using Silvaco TCAD device simulation software. Energy band diagram of the device has been shown with different gate and drain bias. The effect of other physical parameters like radius of the nanowire, different gate metals and gate dielectrics are considered. The current voltage and transconductance characteristics of the device have been studied in details by taking into account Schrodinger-Poisson quantum models. A flat gm-Vg curve proves the linearity of the device in a wide range of gate voltages. All the results agree well with the experimentally available data.

\section{STRUCTURE OF DEICE AND METHODS}

Figure 1 shows the three dimensional view of the device simulated where the metal contacts are made of Gold $(\mathrm{Au})$. The gate dielectric $\mathrm{HfO}_{2}$ is of $2 \mathrm{~nm}$ thickness which is deposited on GaN all around the structure and then the gate metal contact is placed on the dielectric material. The source and drain is doped with $n$-type material with a concentration of $10^{19} / \mathrm{cm}^{3}$ and the channel is $p$-type doped with a concentration of $10^{15} / \mathrm{cm}^{3}$

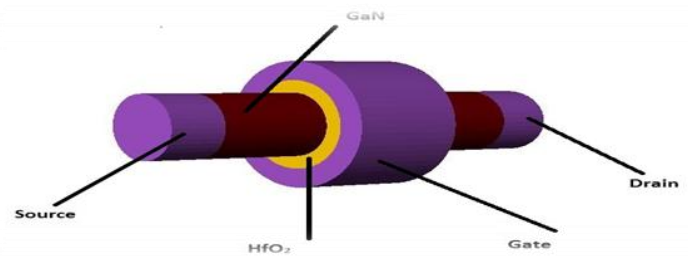

Fig. 1 - 3D structure of the device

There are two main quantum models in Silvaco TCAD: Bohm Quantum Potential and SchrodingerPoisson. We have employed the latter model for the analysis. The simulation employs the Schrödinger model [23] for the simulation coupled with drift-diffusion 
mode space approach. Also field dependent mobility of the charge carriers has been employed along with Shockley-Read Hall recombination parameter.

Table 1 - Summarizes the parameters considered for device simulation

\begin{tabular}{|l|l|}
\hline Parameters Used in Simulation \\
\hline Source/Drain Length & $10 \mathrm{~nm}$ \\
\hline Source/Drain Doping( $n$-type $)$ & $10^{19} / \mathrm{cm}^{3}$ \\
\hline Channel Doping $(p$-type) & $10^{15} / \mathrm{cm}^{3}$ \\
\hline Gate Thickness & $2 \mathrm{~nm}$ \\
\hline
\end{tabular}

The Eigen state energy is given by the following equation:

$$
-\frac{h^{2}}{2} \frac{\partial}{\partial y}\left(\frac{1}{m_{y}^{v}(x, y)} \frac{\partial \psi_{i v}}{\partial y}\right)+E_{c}(x, y) \psi_{i v}=E_{i v} \psi_{i v}
$$

Where the various terms used have their usual meanings.

The two dimensional confinement of electron concentration is given as:

$$
\begin{aligned}
& n(x, y)=2 \frac{\sqrt{\left(2 k_{B} T\right)}}{h} \sum_{v} \sqrt{m_{z}^{v}(x, y)} \times \\
& \times \sum_{i=0}^{\infty}\left|\psi_{i v}(x, y)\right|^{2} F_{-\frac{1}{2}}\left(-\frac{E_{i v}-E_{F}}{k_{B} T}\right)
\end{aligned}
$$

Where $F_{-1 / 2}$ is the Fermi integral of order $-1 / 2$.

\section{RESULTS AND DISCUSSION}

Silvaco TCAD has been used to design and analyze the electrical characteristics of the nanowire FET.

Energy Band Diagram

Figure 2 shows the energy band diagram when the drain voltage is varied from $10 \mathrm{~V}$ to $20 \mathrm{~V}$ at a constant value of $V_{g}(=5 \mathrm{~V})$. When the drain voltage is increased, the density of electron increases on the source side and hence it tends to lower the energy of the conduction band and valence band as lower energy excitation is required to make an electron move from valence band to conduction band.

Figure 3 shows the conduction and valence band when the gate voltage is varied from $1 \mathrm{~V}$ to $5 \mathrm{~V}$. The drain voltage is kept constant at $10 \mathrm{~V}$. The gate voltage is the main controller of the current from the drain to source side (or movement of electrons from source to drain side). As is expected, the potential barrier is reduced when the gate voltage is increased. The band diagram gives us an intuitive explanation of the current surge when gate voltage is increased.

Effect of Radii

The Radii of the GaN Nanowire was varied at $10 \mathrm{~nm}, 15 \mathrm{~nm}$ and $20 \mathrm{~nm}$. The output and transfer characteristics of the device were obtained.

Figure 4 shows the drain current $\left(I_{d}\right)$ versus the drain voltage characteristics at $10 \mathrm{~nm}$ nanowire radius and $10 \mathrm{~nm}$ channel length. The gate voltage $\left(V_{g}\right)$ was kept constant at $5 \mathrm{~V}$ and drain voltage $\left(V_{d}\right)$ was varied from $0 \mathrm{~V}$ to $40 \mathrm{~V}$. It can be observed that the device

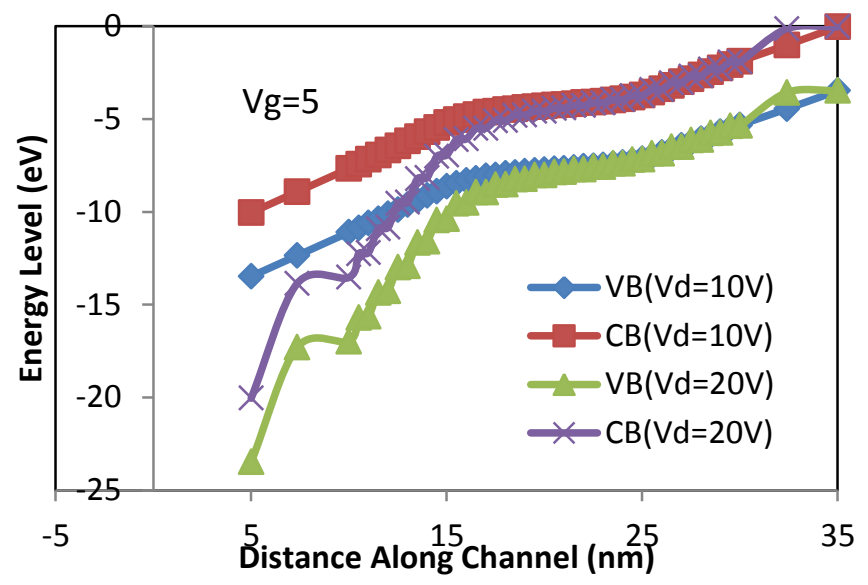

Fig. 2 - Valence and Conduction Band (Variation of Vd from $10 \mathrm{~V}$ to $20 \mathrm{~V}$ ) with $V g=5 \mathrm{~V}$

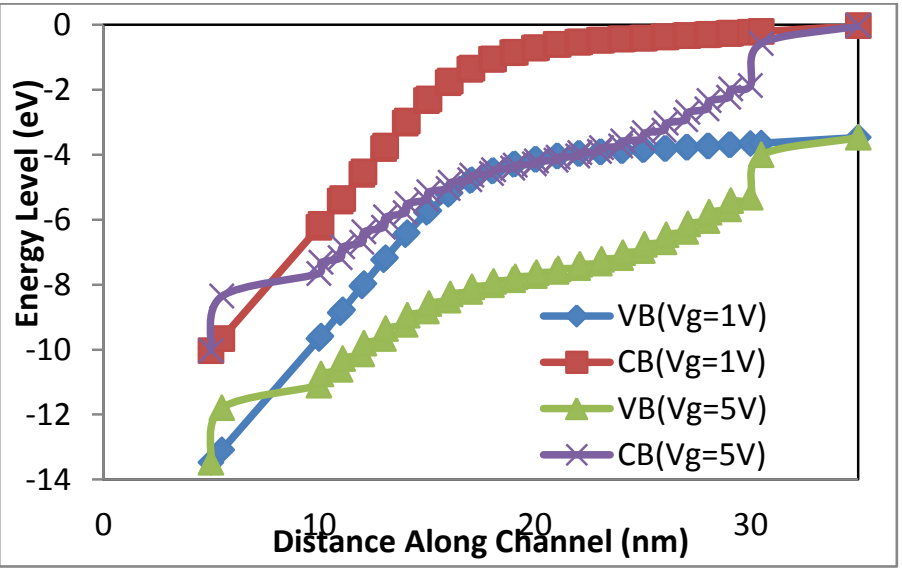

Fig. 3 - Valence and Conduction Band (Variation of $V_{g}$ from $1 \mathrm{~V}$ to $5 \mathrm{~V}$ ) with $V_{d}=10 \mathrm{~V}$

readily works till $40 \mathrm{~V}$. One of the major observation of the GaN NWT is that the drain current remains fairly constant in the saturation region unlike many other device where the channel length modulation factor deviates the working of the short channel devices from the ideal conditions.

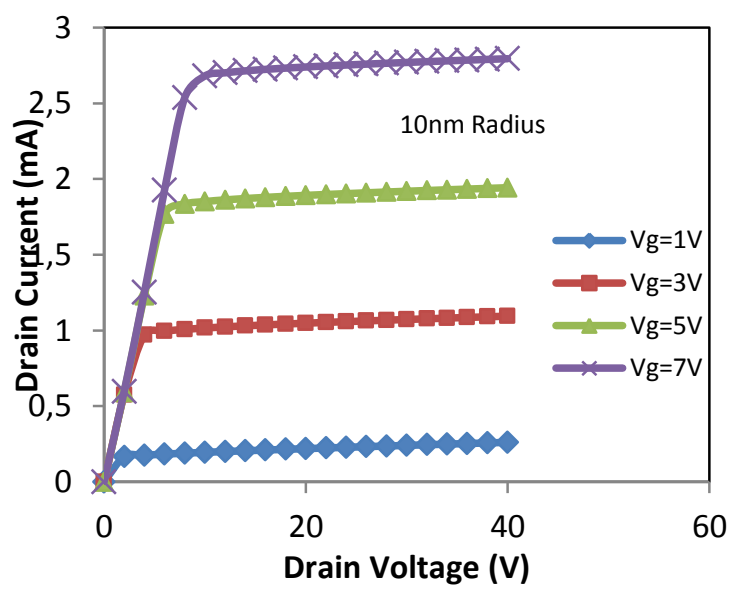

Fig. 4 - Variation of drain current with drain voltage at 10nm radius

Figure 5 shows the variation of the drain current 
with the variation of radius of the device. It can be observed that the drain current is higher at higher values of nanowire radius. The reason for high current at larger radius is quite intuitive. When the radius is large, the electron concentration is more which in turn contributes to the high density of the electrons in the channel formed after applying gate voltage. However, choosing a right combination of nanowire radius and nanowire length, optimized value of drain current can be obtained.

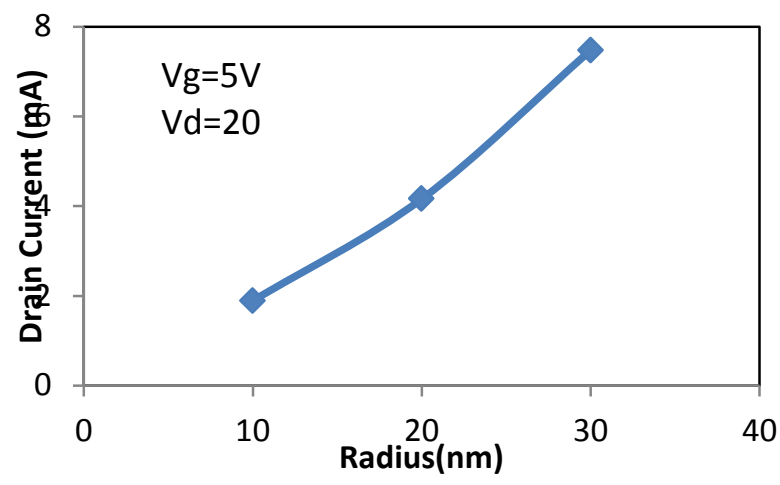

Fig. 5 - Variation of drain current with radius of the nanowire at a constant value of $V_{d}$ and $V_{g}$

Figure 6 shows the $I_{d}$ vs $V_{g}$ characteristics of the device, where the gate voltage has been varied between $0 \mathrm{~V}$ to $10 \mathrm{~V}$ and the drain voltage has been kept constant at $10 \mathrm{~V}$. The reason for keeping the drain voltage at $10 \mathrm{~V}$ is because the device is being considered for high voltage applications. The radii has again been kept at $10 \mathrm{~nm}, 15 \mathrm{~nm}$ and $20 \mathrm{~nm}$. It can be observed that the subthreshold characteristic is better in the case of smaller radius and it becomes worse when the channel radius is increased. This can again be explained by the fact that when the gate voltage is reduced, the electron concentration should decrease at the junction of $\mathrm{GaN}$ and $\mathrm{HfO}_{2}$. But when the radius is increased the control over the channel reduces because the channel is farther away from the central axis of the cylinder.

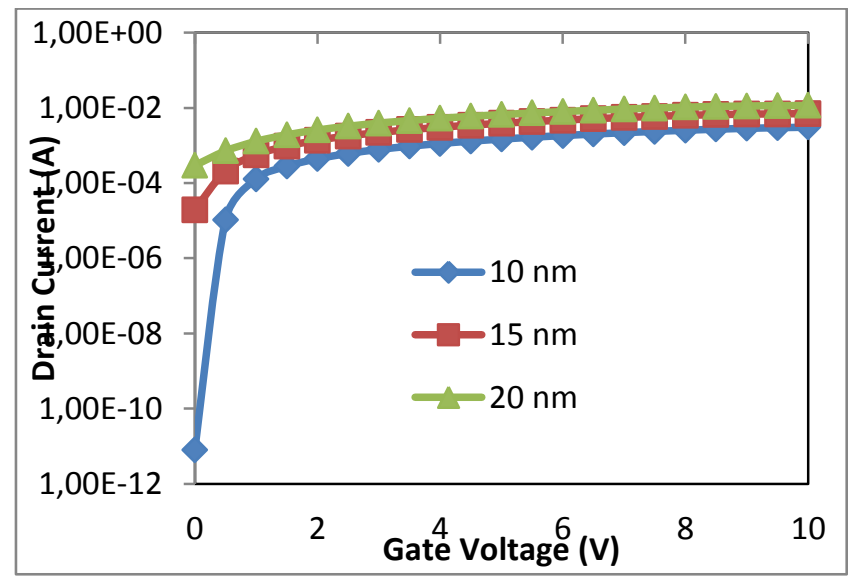

Fig. 6 - $I_{d}$ vs $V_{g}$ for different radii

Effect of Gate Dielectric

The transfer characteristic curves of GaN Nanowire have been plotted in Figure 7 using different gate die- lectric materials. Here, we compare the characteristics using three different gate dielectrics, $\mathrm{SiO}_{2}, \mathrm{Al}_{2} \mathrm{O}_{3}$ and $\mathrm{HfO}_{2}$, the last two being high- $k$ dielectric material. The consideration of high- $k$ dielectric material is very essential because $\mathrm{SiO}_{2}$ becomes a challenging material when scaled down to sizes below $5 \mathrm{~nm}$. So alternatives have to be used which have higher dielectric constants and hence can be scaled down easily and the EOT (Effective Oxide Thickness) can remain the same as before [16-17].

$I_{d}$ vs $V_{g}$ characteristics of the device gives an insight to the switching characteristic of the device when different gate dielectric materials are used. This can give us an understanding as to which material is better and by how much degree it is affecting the operation of the device. As can be observed from Figure 7, the subthreshold slope is lowest in the case of $\mathrm{HfO}_{2}$ and the worst in the case of $\mathrm{SiO}_{2}$. The dielectric constant of $\mathrm{HfO}_{2}$ is nearly $25, \mathrm{Al}_{2} \mathrm{O}_{3}$ is 10 and that of $\mathrm{SiO}_{2}$ is 3.9 . At the same thickness of $2 \mathrm{~nm}, \mathrm{HfO}_{2}$ provides a better current control in the channel since it has good thermal stability, high recrystallization temperature, sound interface quality, low defects and prevents leakage current.

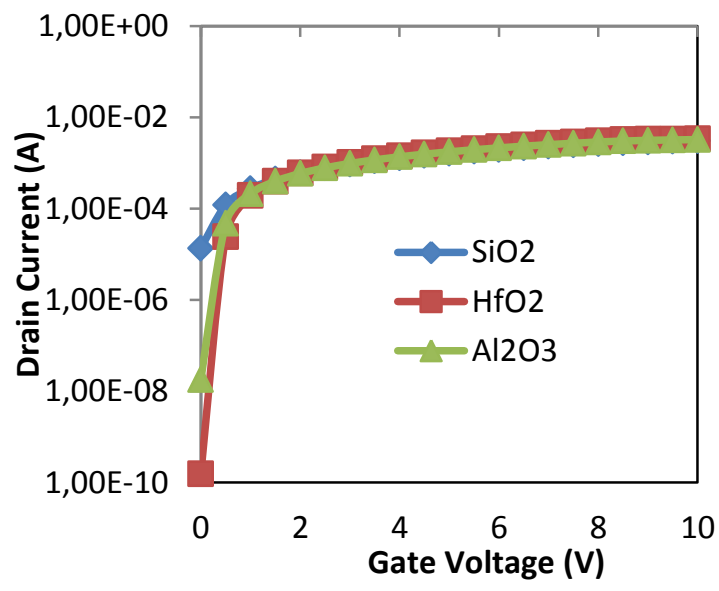

Fig. 7 - $I_{d}$ vs $V_{g}$ for different gate dielectric materials

Effect of Gate Metal

Type of gate metals greatly contribute to the device characteristics. The metals that have been employed for this purpose are Aluminium, Titanium and Gold. Also the use of metal stacks is a common practice. The work function of all these metals is different which gives them their unique properties. The work function of the metal is defined as the energy required to pull out an electron from the Fermi energy level to infinity.

First we consider the $I_{d}$ vs $V_{g}$ characteristics of the device in Figure 8. The drain voltage is kept at $5 \mathrm{~V}$. This high value of $V_{d}$ can actually give us an insight how well the gate metal is able to control the drain current under such conditions. It can be observed from the plot that when $\mathrm{Al}$ is used as the gate there is drain current of order -4 . But when Gold is used as the gate metal the order is reduced to -15 at $V_{g}=0 \mathrm{~V}$.

Figures 8 portray two interesting facets of the gate metal contact. If the gate metal to be used has a greater workfunction, then we have a better $I_{d}$ vs $V_{g}$ characteristic curve which points to the fact that the device is actually off when gate voltage goes below the threshold 
voltage (steeper subthreshold curve indicates lower subthreshold current). But on the other hand, when the drain current vs workfunction is plotted it can be clearly observed that the drain current increases when the workfunction of the gate metal is reduced. So the gate metal is like a trade off factor. Whether we want better switching (steeper subthreshold slope) or better amplification (more drain current), would dictate the choice of gate metal.

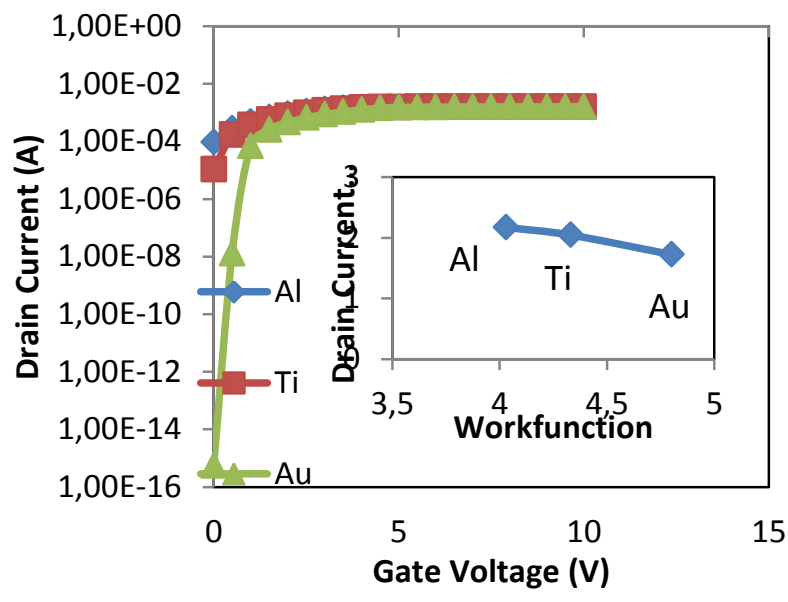

Fig. 8 - $I_{d}$ vs $V_{g}$ for different gate contact metals (inset shows Id vs workfunction of three different gate contact metals)

Effect of Gate Length

Now, the GaN nanowire has been considered at different gate lengths. Gate length is a very important factor for consideration owing to the continuous scaling down of the device. Drain current has been computed for three different devices with $L_{g}=10 \mathrm{~nm}, 20 \mathrm{~nm}$ and $30 \mathrm{~nm}$ respectively. Figure 9 clearly describes the relationship between varying gate lengths and the drain current at a constant gate voltage of $5 \mathrm{~V}\left(V_{g}=5 \mathrm{~V}\right)$.

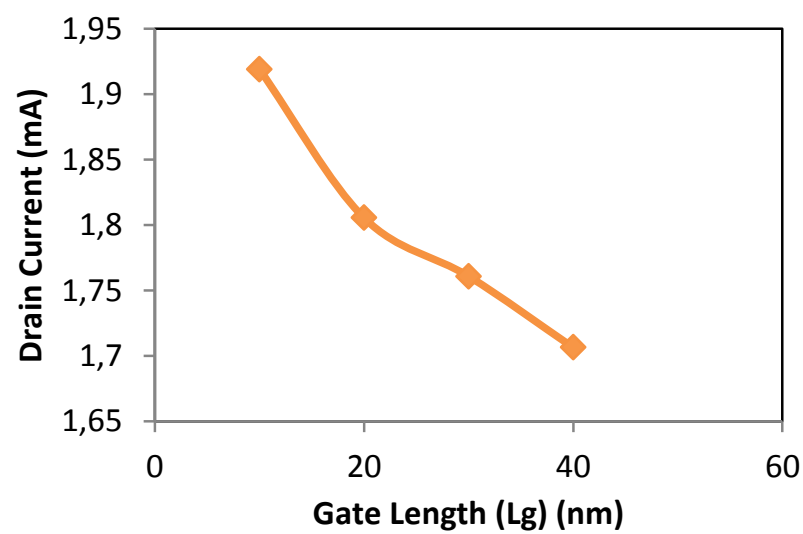

Fig. 9 - Variation of drain current with gate length

The above figure clearly delineates the mentioned relationship. It can be observed that the current decreases with an increase in gate length. One important fact, the slope of the line joining the drain currents at point $10 \mathrm{~nm}$ and $20 \mathrm{~nm}$ is more than that of the lines connecting other three points (where the slope can be construed to be linear). So the current difference at $10 \mathrm{~nm}$ and $20 \mathrm{~nm}$ gate length (about $0.1 \mathrm{~mA}$ ) is more than that of $20 \mathrm{~nm}$ and $30 \mathrm{~nm}$ or $30 \mathrm{~nm}$ and $40 \mathrm{~nm}$ (about $0.05 \mathrm{~mA}$ ). So, reducing the gate length below $10 \mathrm{~nm}$ substantially increases the current. Chances of the device failing at such short gate lengths at even high voltages would be difficult because of high value of band gap.

Device transconductance

Transconductance of a semiconductor device is quite an important characteristic wherein it signifies the amplification capability of the device at a certain value of parameters. Transconductance is given as:

$$
g_{m}=\frac{d I_{d}}{d V_{G S}}
$$

The above equation tells us how much the drain current changes when the input voltage is changed. Higher value of transconductance means that a small change in the input signal, $\mathrm{V}_{\mathrm{GS}}$ brings a large change in the output current, $I_{d}$ (large amplification). Small value of transconductance means smaller amplification capability.

As shown in the Figure 10, drain voltage has been kept constant at $10 \mathrm{~V}$ and gate voltage has been varied between $-2 \mathrm{~V}$ and $10 \mathrm{~V}$ and transconductance has been traced. It can be observed that the amplification property of the device with $L_{g}=10 \mathrm{~nm}$ goes down whereas that of $L_{g}=20 \mathrm{~nm}$ and $30 \mathrm{~nm}$ decreases at a slower rate. It is also seen from the figure that at $L_{g}=10 \mathrm{~nm}$ the maximum value of transconductance is comparable to those at $20 \mathrm{~nm}$ and $30 \mathrm{~nm}$ at lower gate voltages.

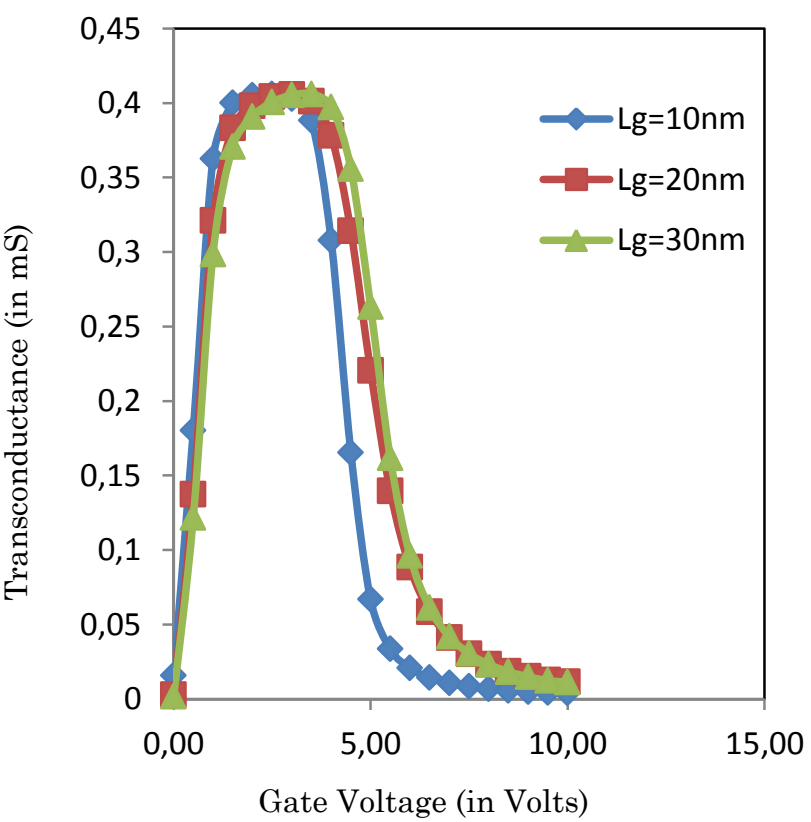

Fig. 10 - Transconductance of the device at three different gate length

Figure 11 shows the transconductance of the device at three different radius. It can be observed that highest transconductance is of the device with greater radius.

As seen from Figure 10 and 11, flatter $g_{m}-V_{g}$ curve results in higher third order intercept point (IIP3) value which indicate better linearity performance of the scaled device. 


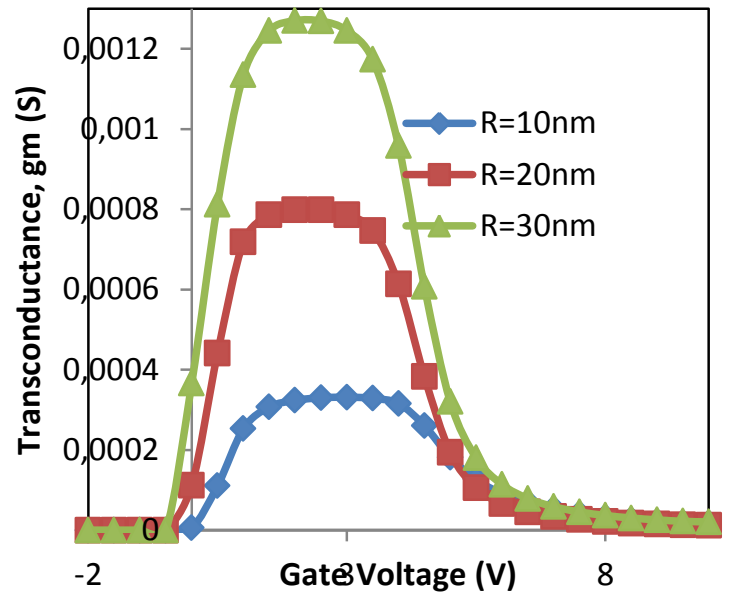

Fig. 11 - Transconductance of the device at three different nanowire radii

Table I shows the comparison of different types of nanowires available

Table II - Comparison of different nanowire transistors
Gate Stack (1 nm/4 nm layers)

The comparison table includes different type of nanowires including $\mathrm{Si}$, Poly-Si, InGaAs and GaN nanowires. Also several types of gate geometries and experimental as well as simulated results have been considered. The important parameters like ON current, subthreshold slope and DIBL have been compared. A high $I_{\text {on }} / I_{\text {off }}$ achieved in the present case show the usability of the GaN nanowire transistor for digital applications

\section{CONCLUSIONS}

Nanoscale GaN transistor has been designed and simulated in the cylindrical coordinate. Gate length of $10 \mathrm{~nm}$ and nanowire radius of $10 \mathrm{~nm}$ has been used in the analysis. At such low dimensions quantum mechanical analysis of the device characteristics has been done. The effect of high $k$ dielectric materials like $\mathrm{HfO}_{2}$ and $\mathrm{Al}_{2} \mathrm{O}_{3}$ has been considered. Also the effect of different metal gates on the drain current of the device has been considered. The device has been operated at high voltages up to $40 \mathrm{~V}$. This justifies the utilization of this nanowire transistor in high voltage applications. The simulation model can further be extended to obtain the capacitance-voltage and small signal characteristics of the GaN nanowire transistor.

\begin{tabular}{|l|l|l|l|l|l|l|l|l|l|}
\hline $\mathrm{X}$ & Ref [24] & Ref [25] & Ref [26] & Ref [27] & Ref [28] & Ref [18] & Ref [18] & This work & This work \\
\hline $\begin{array}{l}\text { Nanowire } \\
\text { type }\end{array}$ & $\mathrm{Si}$ & Poly-Si & $\begin{array}{l}\text { Poly-Si } \\
\text { JNT }\end{array}$ & InGaAs & Si & GaN & GaN & GaN & GaN \\
\hline $\begin{array}{l}\text { NW cross } \\
\text { sectional } \\
\text { type }\end{array}$ & $\begin{array}{l}\text { Rectangular- } \\
\text { like }\end{array}$ & Tri-gate & GAA & Rectangular & $\begin{array}{l}\text { GAA - } \\
\text { circular }\end{array}$ & Triangular & Triangular & $\begin{array}{l}\text { GAA- } \\
\text { cylindrical }\end{array}$ & $\begin{array}{l}\text { GAA- } \\
\text { cylindrical }\end{array}$ \\
\hline$L_{g}(\mathrm{~nm})$ & 65 & 230 & 80 & 50 & 8 & 50 & 20 & 20 & 10 \\
\hline $\begin{array}{l}\text { EOT or } \\
T_{\text {ox }}(\mathrm{nm})\end{array}$ & 3 & 5 & $\mathrm{NA}$ & $5^{*}$ & 4 & 13 & 10 & 2 & 2 \\
\hline$V_{d}($ Volts) & 1 & 1 & 0.1 & 0.5 & 1.2 & 4 & 4 & 4 & 4 \\
\hline $\begin{array}{l}\text { SS } \\
(\mathrm{m} \text { V/dec) }\end{array}$ & 70 & 98 & 61 & 110 & 75 & 91 & 112 & 60 & 75 \\
\hline $\begin{array}{l}\text { DIBL } \\
(\mathrm{mV} / \mathrm{V})\end{array}$ & 62 & $\mathrm{NA}$ & $<10$ & $\mathrm{NA}$ & 22 & 40 & 34 & 20 & 50 \\
\hline$I_{\text {on }} / I_{\text {off }}$ & $\sim 1 \times 10^{6}$ & $\sim 5 \times 10^{4}$ & $\sim 1 \times 10^{7}$ & $\sim 2 \times 10^{3}$ & $\sim 1 \times 10^{6}$ & $\sim 1 \times 10^{8}$ & $\sim 1 \times 10^{8}$ & $\sim 1 \times 10^{8}$ & $>1 \times 10^{8}$ \\
\hline
\end{tabular}

\section{REFERENCES}

1. W. Feng, R. Hettiarachchi, S. Sato, K. Kakushima, M. Niwa, H. Iwai, K. Yamada, K. Ohmori, Jpn. J Appl. Phys. 51(s4), 04DC06 (2012).

2. J. Li, S. Pud, M.S. Vitusevich, Nanotechnology 25, 275302 (2014).

3. K.H. Goh, S. Yadav, K.L. Low, G. Liang, X. Gong, Y.C. Yeo, IEEE Transactions on Electron Devices 63(3), 1027 (2016).

4. A. Yesayan, F. Jazaeri, J. Sallese, IEEE Trans. Electron Devices, 63(3), 1368 (2016).

5. J. Shin, K. Pi, S. Jung, D. Cho, 2016 IEEE 29th International Conference on Micro Electro Mechanical Systems (MEMS), (2016).

6. N. Chatterjee, S. Pandey, Annual IEEE India Conference (INDICON), 2015.

7. N. Chatterjee, K. Chopra, K. Bhatia, S. Pandey, TENCON IEEE Region 10 Conference, (2015).

8. Kyu-Heon Cho, Young-Hwan Choi, Jiyong Lim, MinKoo Han, Phys. Scripta 78(6), 065802 (2008).

9. X. Gang, E. Xu, N. Hashemi, Z. Bo, F.Y. Fu, W. Tung, Chinese Phys. B 21(8), 086105 (2012).

10. V. Kumaresan, L. Largeau, F. Oehler, H. Zhang,
O. Mauguin, F. Glas, N. Gogneau, M. Tchernycheva, J.C. Harmand, Nanotechnology 27(13), 135602 (2016).

11. L. Dimitrocenko, K. Kundzins, A. Mishnev, I. Tale, A. Voitkans, P. Kulis, IOP Conference Series: Materials Science and Engineering 23(1), 012026 (2011).

12. S. Keller, C. Schaake, N.A. Fichtenbaum, C.J. Neufeld, Y.Wu, K. McGroddy, A. David, S.P. DenBaars, C. Weisbuch, J.S. Speck, U.K. Mishra, J. Appl. Phys. 100(5), 054314 (2006).

13. N.P. Reddy, S. Naureen, S. Mokkapati, K. Vora, N. Shahid, F. Karouta, H.H. Tan, C. Jagadish, Nanotechnology 27(6), 065304 (2016).

14. Hyo-Suk Kim, J.R. Kim, Ju-Jin Kim, Jeong-O. Lee, Journal of the Korean Physical Society 61(12), 2100 (2012).

15. Yu. Huang, X. Duan, Yi. Cui, C.M. Lieber, Nano Lett. 2(2), 101 (2002).

16. S.K. Lee, H.K. Seong, K.C. Choi, N.K. Cho, H.J. Choi, E.K. Suh, K.S. Nahm, Silicon carbide and related materials - 2005 : proceedings of the International Conference on Silicon Carbide and Related Materials: Pittsburgh, Pennsylvania, USA : September 18-23 2005, pp.1549-1552, (2006). 
17. M.S. Kang, J.H. Lee, H.S. Lee, S.M. Koo, J Nanosci Nanotechno 13(10), 7042 (2013).

18. Chi-Kang Li, Po-Chun Yeh, Jeng-Wei Yu, Lung-Han Peng, Yuh-Renn Wu, J. Appl. Phys. 114, 163706, (2013).

19. Y.Q. Chen, X.B. Xu, Y.D. Lu, X. Wang, Y.F. Fen, phys. status solidi 212(2), 390 (2015).

20. J. Mussener, J. Teubert, P. Hille, M. Schäfer, J. Schörmann, Maria de la Mata, J. Arbiol, M. Eickhoff, Nano Lett. 14, 5118 (2014).

21. Jum-Wei Zhao, Yue-Fei Zhang, Yong-He Li, ChaoHua Su, Xue-Mei Song, Hui Yan and Ru-Zhi Wang, Sci. Rep. 5, 17692 (2015).

22. Z. Fang, E. Robin, E. Rozas-Jimenez, A. Cros, F. Donatini, N. Mollard, J. Pernot, B. Daudin, Nano Lett. 15(10), 6794 (2015).

23. V. Patnaik, A. Gheedia, M. Kumar, Silvaco.com, 2008. [Online]. Available: [Accessed: 27- Mar- 2016].
24. S. Sato, Y. Lee, K. Kakushima, P. Ahmet, K. Ohmori, K. Natori, K. Yamada, H. Iwai, Proceedings of the European Solid-State Device Research Conference (ESSDERC), (2010).

25. M. Oda, K.S. Yuuichi Kamimuta, M. Saitoh, p. 125, IEDM (2015).

26. Po-Yi Kuo, Jer-Yi Lin, Tien-Sheng Chao, p. 133, IEDM (2015).

27. N. Waldron, S. Sioncke, J. Franco, L. Nyns, A. Vais , X. Zhou, H.C. Lin, G. Boccardi, J.W. Maes, Q. Xie, M. Givens, F. Tang, X. Jiang, E. Chiu, A. Opdebeeck, C. Merckling, F. Sebaai, D. van Dorp, L. Teugels, A. Sibaja Hernandez, K. De Meyer, K. Barla, N. Collaert, Y-V. Thean, IEDM, p.31.1, (2015).

28. Y. Jiang, T.Y. Liow, N. Singh, L.H. Tan, G.Q. Lo, D.S. H. Chan, D.L. Kwong, Tech. Dig. of Symp. VLSI Tech., 34 (2008). 